\title{
On the structure of generalized Appell sequences of paravector valued homogeneous monogenic polynomials
}

\author{
C. Cruz*, M. I. Falcão*`` and H. R. Malonek**** \\ ${ }^{*}$ Center for Research and Development in Mathematics and Applications, University of Aveiro, Portugal \\ ${ }^{\dagger}$ Department of Mathematics and Applications, University of Minho, Portugal \\ ${ }^{* *}$ Department of Mathematics, University of Aveiro, Portugal
}

\begin{abstract}
The fact that generalized Appell sequences of monogenic polynomials in the setting of hypercomplex function theory also satisfy a corresponding binomial type theorem allows to obtain their explicit structure. Recently it has been obtained a complete characterization in the case of paravector valued homogeneous polynomials of three real variables. The aim of this contribution is the study of paravector valued homogeneous polynomials of four real variables, where new types of generalized Appell sequences could be detected.
\end{abstract}

Keywords: homogeneous monogenic polynomials, hypercomplex analysis, Appell sets PACS: 02.30.-f, 02.30.Lt

\section{INTRODUCTION AND BASIC NOTATION}

Let $\left\{e_{1}, e_{2}, \cdots, e_{n}\right\}$ be an orthonormal base of the Euclidean vector space $\mathbb{R}^{n}$ with a product according to the multiplication rules $e_{k} e_{l}+e_{l} e_{k}=-2 \delta_{k l}, k, l=1, \cdots, n$, where $\delta_{k l}$ is the Kronecker symbol. This non-commutative product generates the $2^{n}$-dimensional Clifford algebra $C \ell_{0, n}$ over $\mathbb{R}$ and the set $\left\{e_{A}: A \subseteq\{1, \cdots, n\}\right\}$ with $e_{A}=$ $e_{h_{1}} e_{h_{2}} \cdots e_{h_{r}}, 1 \leq h_{1}<\cdots<h_{r} \leq n, e_{\emptyset}=e_{0}=1$, forms a basis of $C \ell_{0, n}$. The real vector space $\mathbb{R}^{n+1}$ will be embedded in $C \ell_{0, n}$ by identifying the element $\left(x_{0}, x_{1}, \cdots, x_{n}\right) \in \mathbb{R}^{n+1}$ with the element $x=x_{0}+\underline{x} \in \mathscr{A}_{n}:=\operatorname{span}_{\mathbb{R}}\left\{1, e_{1}, \ldots, e_{n}\right\} \subset$ $C \ell_{0, n}$. Here, $x_{0}$ and $\underline{x}=e_{1} x_{1}+\cdots+e_{n} x_{n}$ are, the so-called, scalar and vector parts of the paravector $x \in \mathscr{A}_{n}$. The conjugate of $x$ is $\bar{x}=x_{0}-\underline{x}$ and the norm $|x|$ of $x$ is defined by $|x|^{2}=x \bar{x}=\bar{x} x=x_{0}^{2}+x_{1}^{2}+\cdots+x_{n}^{2}$.

In what follows we consider $C \ell_{0, n}$-valued functions defined in some open subset $\Omega \subset \mathbb{R}^{n+1}$, i.e., functions of the form $f(z)=\sum_{A} f_{A}(z) e_{A}$, where $f_{A}(z)$ are real valued. We suppose that $f$ is hypercomplex differentiable in $\Omega$ in the sense of [1], [2], i.e. has a uniquely defined areolar derivative $f^{\prime}$ in each point of $\Omega$. Then $f$ is real differentiable (even real analytic) and $f^{\prime}$ can be expressed in terms of the partial derivatives with respect to $x_{k}$ as $f^{\prime}=\frac{1}{2}\left(\partial_{0}-\partial_{\underline{x}}\right) f$, where $\partial_{0}:=\frac{\partial}{\partial x_{0}}, \quad \partial_{\underline{x}}:=e_{1} \frac{\partial}{\partial x_{1}}+\cdots+e_{n} \frac{\partial}{\partial x_{n}}$. If now $\bar{\partial}:=\frac{1}{2}\left(\partial_{0}+\partial_{\underline{x}}\right)$ is the usual generalized Cauchy-Riemann differential operator, then, obviously $f^{\prime}=\partial f$. Since in [1] it has been shown that a hypercomplex differentiable function belongs to the kernel of $\bar{\partial}$, i.e. satisfies the property $\bar{\partial} f=0$ ( $f$ is a monogenic function in the sense of Clifford Analysis), then it follows that in fact $f^{\prime}=\partial_{0} f$ like in the complex case.

\section{HYPERCOMPLEX APPELL POLYNOMIALS}

We recall the classical definition of sequences of Appell polynomials [3] adapted to the hypercomplex case: a sequence of monogenic polynomials $\left(\mathscr{F}_{k}\right)_{k \geq 0}$ of exact degree $k$ is called a generalized Appell sequence if $\mathscr{F}_{0}(x) \equiv 1$ and $\mathscr{F}_{k}^{\prime}=k \mathscr{F}_{k-1}, k=1,2, \ldots$ (see e.g. [4]). Another equivalent definition of Appell polynomials is provided by the following result ([5]):

Theorem 1 A monogenic polynomial sequence $\left(\mathscr{F}_{k}\right)_{k \geq 0}$ is an Appell set if and only if it satisfies the binomial-type expansion

$$
\mathscr{F}_{k}(x)=\mathscr{F}_{k}\left(x_{0}+\underline{x}\right)=\sum_{s=0}^{k}\left(\begin{array}{l}
k \\
s
\end{array}\right) \mathscr{F}_{k-s}\left(x_{0}\right) \mathscr{F}_{s}(\underline{x}), x \in \mathscr{A}_{n} .
$$


Example 1 Standard Appell polynomials: ( [4, 5, 6])

$$
\mathscr{P}_{k}\left(x_{0}, \underline{x}\right)=\sum_{s=0}^{k}\left(\begin{array}{l}
k \\
s
\end{array}\right) c_{s}(n) x_{0}^{k-s} \underline{x}^{s}
$$

where

$$
c_{s}(n)=\frac{s ! !(n-2) ! !}{(n+s-1) ! !}, \quad \text { if s is odd, } \quad c_{s}(n)=c_{s-1}(n), \quad \text { if } s \text { is even } .
$$

Example 2 Pseudo-complex polynomials: $([7,8])$

$$
\mathscr{P}_{k}\left(x_{0}, \underline{x}\right)=\sum_{s=0}^{k}\left(\begin{array}{l}
k \\
s
\end{array}\right) x_{0}^{k-s}\left(\left(i_{1} x_{1}+\cdots+i_{n} x_{n}\right)\left(i_{1} e_{1}+\cdots+i_{n} e_{n}\right)\right)^{s}=\left(x_{0}+\left(i_{1} x_{1}+\cdots+i_{n} x_{n}\right)\left(i_{1} e_{1}+\cdots+i_{n} e_{n}\right)\right)^{k},
$$

where $i_{1}, \ldots, i_{n}$ are real parameters such that $i_{1}^{2}+\cdots+i_{n}^{2}=1$.

These two examples play an important role in the present context, as the following recent result in [8] shows.

Theorem 2 In $\mathbb{R}^{3}$, there are exactly two different types of non-trivial Appell polynomials of the form

$$
\mathscr{P}_{k}\left(x_{0}, x_{1}, x_{2}\right)=\sum_{s=0}^{k}\left(\begin{array}{l}
k \\
s
\end{array}\right) d_{s} x_{0}^{k-s}\left(X_{1}\left(x_{1}, x_{2}\right) e_{1}+X_{2}\left(x_{1}, x_{2}\right) e_{2}\right)^{s},
$$

where $X_{j}=X_{j}\left(x_{1}, x_{2}\right), j=1,2$, are two real valued linear functions $\left(\partial_{1} X_{1} \cdot \partial_{2} X_{2} \neq 0\right)$ and $d_{0}=1$ :

1. The 3D standard Appell polynomials (1), corresponding to the case $\partial_{1} X_{2}=\partial_{2} X_{1}=0$.

2. The $3 D$ pseudo-complex polynomials (3), when $\partial_{1} X_{2}=\partial_{2} X_{1} \neq 0$.

Observe that, if $\partial_{1} X_{1} \cdot \partial_{2} X_{2}=0$, then (4) corresponds to trivial Appell polynomials, i.e.

$$
\mathscr{P}_{k}\left(x_{0}, x_{2}\right)=\sum_{s=0}^{k}\left(\begin{array}{l}
k \\
s
\end{array}\right) x_{0}^{k-s}\left(x_{2} e_{2}\right)^{s}=\left(x_{0}+x_{2} e_{2}\right)^{k} \quad \text { or } \quad \mathscr{P}_{k}\left(x_{0}, x_{1}\right)=\sum_{s=0}^{k}\left(\begin{array}{l}
k \\
s
\end{array}\right) x_{0}^{k-s}\left(x_{1} e_{1}\right)^{s}=\left(x_{0}+x_{1} e_{1}\right)^{k},
$$

where we recognize two copies of the ordinary complex power function, namely $z^{k}=(x+i y)^{k}$ after setting $x_{0}:=x$ and $e_{j}:=i, x_{j}:=y, j=1,2$. These cases are trivial in the sense that $e_{1} \partial_{1}+e_{2} \partial_{2}+e_{3} \partial_{3}$ acts only as differential operator with respect to $x_{1}$ or $x_{2}$ if the components of $X$ are not depending on $x_{2}$ or $x_{1}$, respectively. More details about Appell sequences in hypercomplex context and the contributions of other authors to this subject can be found in $[8,9]$.

\section{D HOMOGENEOUS POLYNOMIALS WITH BINOMIAL EXPANSION}

We extend now the result of Theorem 2 , by considering $n=3$ and general paravector valued homogeneous polynomials of the form

$$
\mathscr{P}_{k}\left(x_{0}, x_{1}, x_{2}, x_{3}\right)=\sum_{s=0}^{k}\left(\begin{array}{l}
k \\
s
\end{array}\right) d_{s} x_{0}^{k-s} \underline{X}^{s},
$$

where $\underline{X}:=X_{1}\left(x_{1}, x_{2}, x_{3}\right) e_{1}+X_{2}\left(x_{1}, x_{2}, x_{3}\right) e_{2}+X_{3}\left(x_{1}, x_{2}, x_{3}\right) e_{3}$ and $X_{j}=X_{j}\left(x_{1}, x_{2}, x_{3}\right), j=1,2,3$, are real valued linear functions.

Our purpose here is to choose the linear functions $X_{j}(j=1,2,3)$ and the real coefficients $d_{s}(s=1,2, \ldots)$ in such a way that the corresponding polynomials (5) are monogenic. Since left monogenic functions of the form (5) are also right monogenic, it is enough to solve the real system of first order partial differential equations corresponding to $\bar{\partial} \mathscr{P}_{k}=0$. The use of Theorem 1 allows to conclude that this procedure leads to a characterization of the set of paravector valued homogeneous monogenic polynomials with the Appell-property.

Following [8], we use the normalization condition $d_{0}=1$ in order to keep the ordinary real Appell sequence as the restriction on the real line. Therefore, the first degree polynomial in the sequence (5) is $\mathscr{P}_{1}\left(x_{0}, x_{1}, x_{2}, x_{3}\right)=x_{0}+d_{1} \underline{X}$. and it is clear that such polynomial is monogenic if the real linear functions are of the form

$$
X_{1}=a_{1} x_{1}+\lambda_{1} x_{2}+\lambda_{2} x_{3}, \quad X_{2}=\lambda_{1} x_{1}+a_{2} x_{2}+\lambda_{3} x_{3}, \quad X_{3}=\lambda_{2} x_{1}+\lambda_{3} x_{2}+a_{3} x_{3}
$$


and the coefficient $d_{1}$ is given by

$$
d_{1}=\frac{1}{a_{1}+a_{2}+a_{3}}, \quad \text { for } \quad a_{1}+a_{2}+a_{3} \neq 0 .
$$

We point out that the special cases

$$
\lambda_{1}=\lambda_{2}=a_{1}=0 \quad \text { or } \quad \lambda_{1}=\lambda_{3}=a_{2}=0 \quad \text { or } \quad \lambda_{2}=\lambda_{3}=a_{3}=0
$$

correspond to 3D polynomials and therefore Theorem 2 holds, i.e. (5) are, in fact, 3D standard Appell polynomials, 3D pseudo-complex polynomials or complex powers. Our interest lies, of course, on the other cases. We proceed now by examining under what conditions the second degree polynomial $\mathscr{P}_{2}\left(x_{0}, x_{1}, x_{2}, x_{3}\right)=x_{0}+2 d_{1} \underline{X}+d_{2} \underline{X}^{2}$, with $d_{1}$ given by (6) is a monogenic polynomial. This problem is equivalent, for independent $x_{1}, x_{2}$ and $x_{3}$, to solve the nonlinear system

$$
\left\{\begin{array}{l}
a_{1}-d_{2}\left(a_{1}^{2}+\lambda_{1}^{2}+\lambda_{2}^{2}\right)\left(a_{1}+a_{2}+a_{3}\right)=0 \\
a_{2}-d_{2}\left(\lambda_{1}^{2}+a_{2}^{2}+\lambda_{3}^{2}\right)\left(a_{1}+a_{2}+a_{3}\right)=0 \\
a_{3}-d_{2}\left(\lambda_{2}^{2}+\lambda_{3}^{2}+a_{3}^{2}\right)\left(a_{1}+a_{2}+a_{3}\right)=0 \\
\lambda_{1}-d_{2}\left(\lambda_{1}\left(a_{1}+a_{2}\right)+\lambda_{2} \lambda_{3}\right)\left(a_{1}+a_{2}+a_{3}\right)=0 \\
\lambda_{2}-d_{2}\left(\lambda_{2}\left(a_{1}+a_{3}\right)+\lambda_{1} \lambda_{3}\right)\left(a_{1}+a_{2}+a_{3}\right)=0 \\
\lambda_{3}-d_{2}\left(\lambda_{3}\left(a_{2}+a_{3}\right)+\lambda_{1} \lambda_{2}\right)\left(a_{1}+a_{2}+a_{3}\right)=0
\end{array}\right.
$$

Cumbersome, but straightforward calculations lead to the following cases:

1. $\lambda_{1}=\lambda_{2}=\lambda_{3}=0$

We can assume that $a_{1} a_{2} a_{3} \neq 0$, since the trivial case where one of the $a_{i}$ 's is zero was already considered in (7). The solution of (8) is:

$$
a_{1}=a_{2}=a_{3}=\alpha(\alpha \neq 0) \quad \text { and } \quad d_{2}=\frac{1}{3 \alpha^{2}} .
$$

Since in this case, $d_{1}=\frac{1}{3 \alpha}$, we obtain

$$
\underline{X}=\alpha\left(x_{1} e_{1}+x_{2} e_{2}+x_{3} e_{3}\right) \quad \text { and } \quad \mathscr{P}_{1}(x)=x_{0}+\frac{1}{3} \underline{x} .
$$

Applying Theorem 2 of [6], it is clear that

$$
d_{k}=\frac{c_{k}(3)}{\alpha^{k}},
$$

where $c_{k}(3)$ are the coefficients (2), for the dimension $n=3$. In other words, this case corresponds to the standard Appell polynomials (cf (1)) in $\mathbb{R}^{4}$.

2. $\lambda_{1} \neq 0$ or $\lambda_{2} \neq 0$ or $\lambda_{3} \neq 0$

These cases mean that we assume that either one of the parameters is nonzero and the other two vanish or all the parameters are nonzero. The situation where two of the parameters are nonzero and the other one is zero is not possible (see equations 4-6 of (8)). System (8) has now two different solutions:

i. For the first one we have that

$$
d_{2}=\frac{1}{\left(a_{1}+a_{2}+a_{3}\right)^{2}}=d_{1}^{2}
$$

and $\lambda_{1}, \lambda_{2}, \lambda_{3}$ are roots of

$$
\lambda_{1}^{2}=a_{1} a_{2}, \quad \lambda_{2}^{2}=a_{1} a_{3}, \quad \lambda_{3}^{2}=a_{2} a_{3},
$$

chosen so that $\lambda_{1} \lambda_{2} \lambda_{3}=a_{1} a_{2} a_{3}$. We point out that, due to (9), if $a_{1}, a_{2}$ and $a_{3}$ are nonzero, then they must have the same sign. Following the technique of ([8]), we introduce now the real parameters

$$
i_{1}^{2}:=\frac{a_{1}}{a_{1}+a_{2}+a_{3}}, \quad i_{2}^{2}:=\frac{a_{2}}{a_{1}+a_{2}+a_{3}}, \quad i_{3}^{2}:=\frac{a_{3}}{a_{1}+a_{2}+a_{3}},
$$

which allow to write

$$
\mathscr{P}_{1}(x)=x_{0}+\left(i_{1} x_{1}+i_{2} x_{2}+i_{3} x_{3}\right)\left(i_{1} e_{1}+i_{2} e_{2}+i_{3} e_{3}\right),
$$


for a chosen triplet of those roots $i_{1}, i_{2}$ and $i_{3}$ as defined in (10). Notice that from this relation we obtain $i_{1}^{2}+i_{2}^{2}+i_{3}^{2}=1$ and it follows immediately that $\left(i_{1} e_{1}+i_{2} e_{2}+i_{3} e_{3}\right)^{2}=-1$. This shows the isomorphism of the structure of $\mathscr{P}_{k}(x)$ with $z^{k}$ and it implies also that $\mathscr{P}_{k}(x)=\left(\mathscr{P}_{1}(x)\right)^{k}$. In other words, we obtain the 4D-version of the pseudo-complex polynomials (3), if $\lambda_{1} \lambda_{2} \lambda_{3} \neq 0$ and the $3 \mathrm{D}$-version, in the other cases.

(ii) System (8) also admits another solution, namely

$$
d_{2}=\frac{2}{\left(a_{1}+a_{2}+a_{3}\right)^{2}}=2 d_{1}^{2}
$$

and $\lambda_{1}, \lambda_{2}, \lambda_{3}$ are roots of

$$
\lambda_{1}^{2}=\frac{1}{4} A_{1} A_{2}, \quad \lambda_{2}^{2}=\frac{1}{4} A_{1} A_{3}, \quad \lambda_{3}^{2}=\frac{1}{4} A_{2} A_{3},
$$

where $A_{1}=-a_{1}+a_{2}+a_{3}, A_{2}=a_{1}-a_{2}+a_{3}$ and $A_{3}=a_{1}+a_{2}-a_{3}$ are real parameters which must have the same sign, if none of them vanishes. In this case, the roots $\lambda_{i}$ must be chosen in order to verify the relation $-8 \lambda_{1} \lambda_{2} \lambda_{3}=A_{1} A_{2} A_{3}$. We proceed by adapting the procedure used in case $\mathbf{2}$.i. More precisely, we define the real parameters

$$
j_{1}^{2}:=\frac{A_{1}}{A_{1}+A_{2}+A_{3}}, \quad j_{2}^{2}:=\frac{A_{2}}{A_{1}+A_{2}+A_{3}}, \quad j_{3}^{2}:=\frac{A_{3}}{A_{1}+A_{2}+A_{3}},
$$

which verify the relation $j_{1}^{2}+j_{2}^{2}+j_{3}^{2}=1$. Denoting by $\underline{\tilde{X}}$ the pseudo-complex first degree polynomial

$$
\underline{\tilde{X}}:=\left(j_{1} x_{1}+j_{2} x_{2}+j_{3} x_{3}\right)\left(j_{1} e_{1}+j_{2} e_{2}+j_{3} e_{3}\right),
$$

we can write, after some manipulation,

$$
\underline{X}=\frac{1}{2 d_{1}}(\underline{x}-\underline{\tilde{X}}) \quad \text { and } \quad \mathscr{P}_{1}\left(x_{0}, x_{1}, x_{2}, x_{3}\right)=x_{0}+\frac{1}{2}(\underline{x}-\underline{\tilde{X}}) .
$$

Repeating the same process for $\mathscr{P}_{3}, \mathscr{P}_{4}, \ldots$, we conclude that

$$
d_{s}=\left(\begin{array}{c}
s \\
\left\lfloor\frac{s}{2}\right\rfloor
\end{array}\right) d_{1}^{s}, \quad s=0,1, \ldots,
$$

where $\lfloor\cdot\rfloor$ is the floor function. Therefore a new type of Appell polynomial is obtained:

$$
\mathscr{P}_{k}(x)=\sum_{s=0}^{k}\left(\begin{array}{l}
k \\
s
\end{array}\right)\left(\begin{array}{c}
s \\
\left\lfloor\frac{s}{2}\right\rfloor
\end{array}\right) x_{0}^{k-s}\left(d_{1} \underline{X}\right)^{s}=\sum_{s=0}^{k}\left(\begin{array}{l}
k \\
s
\end{array}\right)\left(\begin{array}{c}
s \\
\left\lfloor\frac{s}{2}\right\rfloor
\end{array}\right) x_{0}^{k-s} \frac{1}{2^{s}}(\underline{x}-\underline{\tilde{X}})^{s}=\sum_{s=0}^{k}\left(\begin{array}{l}
k \\
s
\end{array}\right) x_{0}^{k-s} c_{s}(2)(\underline{x}-\underline{\tilde{X}})^{s} .
$$

\section{ACKNOWLEDGMENTS}

This work was supported by FEDER founds through COMPETE-Operational Programme Factors of Competitiveness ("Programa Operacional Factores de Competitividade") and by Portuguese funds through the Center for Research and Development in Mathematics and Applications (University of Aveiro) and the Portuguese Foundation for Science and Technology ("FCT-Fundação para a Ciência e a Tecnologia"), within project PEst-C/MAT/UI4106/2011 with COMPETE number FCOMP-01-0124-FEDER-022690. The research of the first author was also supported by FCT under the fellowship SFRH/BD/44999/2008.

\section{REFERENCES}

1. K. Gürlebeck, and H. Malonek, Complex Variables Theory Appl. 39, 199-228 (1999).

2. H. Malonek, Complex Variables Theory Appl. 14, 25-33 (1990).

3. P. Appell, Ann. Sci. École Norm. Sup. 9, 119-144 (1880).

4. M. I. Falcão, and H. Malonek, Generalized exponentials through Appell sets in $\mathbb{R}^{n+1}$ and Bessel functions, in AIP Conference Proceedings, edited by T. E. Simos, G. Psihoyios, and C. Tsitouras, 2007, vol. 936, pp. 738-741.

5. I. Cação, M. I. Falcão, and H. Malonek, Math. Comput. Model. 53, 1084-1094 (2011).

6. I. Cação, M. I. Falcão, and H. Malonek, Comput. Methods Funct. Theory 12, 371-391 (2012).

7. I. Cação, and H. Malonek, On Complete Sets of Hypercomplex Appell Polynomials, in AIP Conference Proceedings, edited by T. E. Simos, G. Psihoyios, and C. Tsitouras, 2008, vol. 1048, pp. 647-650.

8. H. Malonek, and M. I. Falcão, Advances in Applied Clifford Algebras, (2012) doi:10.1007/s00006-012-0361-5.

9. S. Bock, and K. Gürlebeck, Math. Methods Appl. Sci. 33, 394-411 (2010). 